\title{
A Study of Microbial Colonization and their Antibiotic Resistance Pattern in Endotracheal Aspirate Cultures in Intensive Care Unit Patients at a Tertiary Care Hospital of Eastern UP, India
}

\author{
Vandana Upadhyay, Ankur Kumar*, Jayesh, Amresh Kumar Singh \\ Department of Microbiology, BRD Medical College Gorakhpur, U.P., India \\ *Corresponding author
}

\begin{abstract}
A B S T R A C T
Nosocomial infections are a critical issue among intubated patients. Respiratory tract infection in relation to intubation and/ or mechanical ventilation has been reported to vary from $4 \%$ to $28 \%$ and this rate has been thought to be 21 times higher than in patients

Keywords

Endotracheal tube,

Ventilator-associated

pneumonia, Nosocomial

infection

Article Info

Accepted:

20 March 2018

Available Online:

10 April 2018

without endotracheal tube. The aim of our study was to identify the pathogens isolated from culture of endotracheal tube aspirates and determine their antibiogram. Patients on mechanical ventilation (MV) for $>48 \mathrm{~h}$ in the intensive care units (ICU), critical care unit (CCU), PICU and NICU were included in this study. All the endotracheal aspirate samples were transported to the Microbiology laboratory for culture and sensitivity testing. In our study we observed a total of 101 ETAs culture positive isolates. The most common microorganism was Klebseilla pneumoniae $28(27 \%)$ and in Gram positive it was CONS 12 $(12 \%)$. The highest resistance was found in $3^{\text {rd }}$ generation cephalosporins and Penicillin group antibiotics, the least in carbapenems and polymyxin B. The key is early identification of the causative microbial agents, antibiotic sensitivity and administration of selected antibiotics. Our observations provide useful information regarding such respiratory infection. Thus ET tip cultures could be useful adjunct to the ATS strategy for ensuring appropriate treatment of intubated patients and in context of a bundled approach to the prevention of ET tip infections.
\end{abstract}

\section{Introduction}

Nosocomial infections are the leading cause of mortality and morbidity among the patients admitted in ICU in the present time. One of the most important types of this infection is pneumonia which commonly occurs in relation to the endotracheal tube and mechanical ventilation named ventilator associated pneumonia. Ventilator-associated pneumonia (VAP) is the most frequent intensive care unit (ICU)-acquired infection, occurring in $9-24 \%$ of patients intubated for longer than 48 hours (Tandia et al., 2015). Nosocomial pneumonia represents approximately one quarter of all nosocomial infections and tracheal intubation increases the risk of infection from 6 to 20 times (American Thoracic Society, 2005; Ferreira et al., 2016). In the literature, the incidence of respiratory tract infection in relation to intubation and/ or mechanical ventilation has been reported to 
vary from $4 \%$ to $28 \%$ and this rate has been thought to be 21 times higher than in patients without endotracheal tube (Diaz et al., 2008; Chastre and Fagon, 2002; Fortaleza et al., 2009; Chevret et al., 1993; Haley et al., 1981).

Patients with mechanical ventilation have an increased risk for respiratory tract infection because the tube which has been inserted in trachea reduces the clearance of bacteria and increases the leakage of secretion around the cuff of tube and disable the cilliary tract by damaging it (Tandia et al., 2015; Ferreira et al., 2016; Diaz et al., 2008). Microbial adhesion on the tube itself results in biofilm formation is one of the major cause of infection. In long-term ventilated patients subglottic secretions can accumulate above the cuff of the ETT and hence present as an ideal growth medium for bacteria (Haley et al., 1981; https://www.bactiguard.se/en/our products/endotracheal-tube).

By micro-aspiration along the cuff, these contaminated secretions might pass into the lower respiratory tract and become a potential cause of lower airway-infection including VAP (https://www.bactiguard.se/en/our products/endotracheal-tube). Artificial airways generally are colonized with potentially pathogenic microbes. Colonizing bacteria may arise from the upper airway, ventilator tubing or reservoirs (Depuydt et al., 2006). Lower respiratory tract infections in intubated patients include ventilator-associated tracheobronchitis (VAT) and ventilatorassociated pneumonia (VAP), these infections are increasingly caused by multidrug-resistant bacteria, which colonize the patient's oropharynx and enter the lower respiratory tract around the endotracheal tube cuff or through the lumen. Progression of colonization to VAT and, in some patients, to VAP is related to pathogenesis and virulence of invading bacteria (Donald et al., 2010).
Over the past decade, the incidence of lower respiratory tract infection due to multidrug resistant (MDR) pathogens, such as methicillin-resistant Staphylococcus aureus (MRSA) and gram-negative bacilli (eg, Klebsiella pneumonia, Escherichia coli, Pseudomonas aeruginosa, and Acinetobacter baumanni i) has been increasing (Chevret et al., 1993). Understanding the pathogenesis of VAT (ventilator- associated tracheobronchitis) and VAP (ventilator associated pneumonia) is crucial for establishing principles for therapy and prevention. Hence intubation with mechanical ventilation increases the risk of bacterial pneumonia (Chevret et al., 1993; Nseir et al., 2008).

Widespread use of antibiotics in intensive careunits is a potential cause of the emergence of nosocomial infections caused by antibioticresistant bacteria (Kollef and Fraser, 2001; Chandra Mouli et al., 2016). Current guidelines for the management of VAP strongly recommend the use of early, appropriate empirical antibiotic therapy based on patient risk factors for multidrug-resistant pathogens (Chevret et al., 1993; Kollef and Fraser, 2001; Chandra Mouli et al., 2016).

Routine endotracheal aspirate cultures of critically ill patients in ICUs may be predictive of patients who are at high risk of invasive disease, and may guide the selection of appropriate empirical therapy based on the predominant pathogens identified in microbial cultures in the event of the development of VAP (Joseph et al., 2010).

The decision regarding choice of antibiotic has to be based on microbiological culture and sensitivity pattern. Therefore the aim of our study was to identify the pathogens from culture of endotracheal tube and determine the risk factors of disease and their antibiogram in patients admitted in tertiary care hospital of Eastern UP. 


\section{Materials and Methods}

This was an Observational Cross- Sectional study of 1 year period from June 2016 to May 2017 conducted at Department of Microbiology, BRD Medical College, Gorakhpur.

All the patients on mechanical ventilation (MV) for $>48 \mathrm{~h}$ in the different intensive care units (ICU), critical care unit (CCU) and PICU, NICU of BRD Medical college and hospital were included in this study. Patients with pneumonia prior to MV or within $48 \mathrm{~h}$ of MV were excluded.

The following data was collected from the patients enrolled in the study including age, gender, and underlying illness with all clinical information, duration of hospitalization, duration of mechanical ventilation and details of prior antibiotic therapy.

All the endotracheal aspirate samples were transported to the Microbiology laboratory and immediately inoculated on Blood agar, MacConkey agar and chocolate agar. The culture plates were incubated at $37^{\circ} \mathrm{C}$ for 48 hours before issuing a negative result. Any growth of microorganism on the culture plates was identified by colony characteristics, Gram staining and standard biochemical tests (Meligy et al., 2015; Collee et al., 1996). Bacterial concentrations (cfu/mL) were calculated. Microorganisms with counts > $10^{4} \mathrm{cfu} / \mathrm{mL}$ were submitted for identification and antimicrobial susceptibility testing (Collee et al., 1996; Clinical Laboratory Standard Institute, 2006).

Antibiotic susceptibility of microorganisms isolated from quantitative endotracheal aspirate (ETA) cultures was carried out using the Kirby-Bauer disk diffusion method, on Mueller-Hinton (Hi-media) agar plates by using the commercially available antibiotic disks from Hi-Media (Mumbai, India) as per the Clinical and Laboratory Standard Institute (CLSI) guidelines (Clinical Laboratory Standard Institute, 2006; Clinical Laboratory Standard Institute, 2010).

The following antibiotics disk were used for Gram positive organisms - Ampicillin Sulbactam (10 units), Vancomycin (30 $\mu \mathrm{g})$,

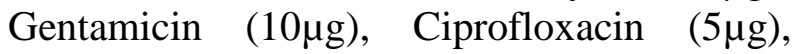
Penicillin G $(10 \mu \mathrm{g})$, Linezolid $(10 \mu \mathrm{g})$, Piperacillin/Tazobactam $\quad(100 / 10 \quad \mu \mathrm{g})$, Imipenem $(10 \mu \mathrm{g})$, Cefotaxime $(30 \mu \mathrm{g})$, Cotrimoxazole $(5 \mu \mathrm{g})$, Clindamycin $(10 \mu \mathrm{g})$, Teicoplanin $(30 \mu \mathrm{g})$, Chloramphenicol (30 $\mu \mathrm{g})$, Erythromycin $(15 \mu \mathrm{g})$ and Cefoxitin $(30 \mu \mathrm{g})$ for MRSA.

For Gram negative organisms, the following antibiotics were used - Amoxicillin $(30 \mathrm{mcg})$, Ofloxacin $(5 \mu \mathrm{g})$, Imipenem $(10 \mu \mathrm{g})$, Meropenem $(10 \mu \mathrm{g})$, Ciprofloxacin $(5 \mu \mathrm{g})$, Ceftazidime $(30 \mu \mathrm{g})$, Cefotaxime $(30 \mu \mathrm{g})$, Ceftriaxone $(30 \mu \mathrm{g})$, Tigecycline $(10 \mu \mathrm{g})$, Tobramycin $(10 \mu \mathrm{g})$, Gentamicin $(10 \mu \mathrm{g})$, Colistin (10 $\mu \mathrm{g}), \quad$ Piperacillin/Tazobactam $(100 / 10 \mu \mathrm{g})$, Chloramphenicol $(30 \mu \mathrm{g})$ and Cotrimoxazole.

Quality control: Reference strains E. coli (ATCC 25922), P. aeruginosa (ATCC 27953) and $S$. aureus (ATCC 25923) were used as control reference strains for identification and drug susceptibility testing (Clinical Laboratory Standard Institute, 2010; Jasuja et al., 2017).

Detection of methicillin resistance in Staphylococcus: Staphylococcus species were further screened for methicillin resistance by using $30 \mu \mathrm{g}$ cefoxitin disc by modified Kirby Bauer disc diffusion method as per the standard guidelines (Jasuja et al., 2017). If the zone diameter $<21 \mathrm{~mm}$, then it was considered methicillin resistant $S$. aureus (MRSA) and methicillin resistant CoNS (MR-CoNS) if < $24 \mathrm{~mm}$. 
Detection of Extended Spectrum BetaLactamase production (ESBL) in Gram negative bacilli: Disk-diffusion method for ESBL screening was performed by using cefpodoxime, ceftazidime, cefotaxime and ceftriaxone, and the isolates showing zone of inhibition to Ceftriaxone $<25 \mathrm{~mm}$, Cefotaxime $<27 \mathrm{~mm}$, Cefpodoxime $<17 \mathrm{~mm}$ and Ceftazidime $<22 \mathrm{~mm}$ in Mueller Hinton Agar (Hi-Media Laboratories, Mumbai, India) for ESBL detection. Phenotypic confirmatory test for ESBL producers was done by both combined disc test (CDT) and double-disc synergy test (DDST) for all isolates that were screened positive, following CLSI guidelines. The confirmatory test was done by potentiated disc diffusion method using ceftazidime alone on one end and ceftazidime with clavulanic acid kept $20 \mathrm{~mm}$ apart. An increase in the zone of inhibition of equal or more than $5 \mathrm{~mm}$ around the ceftazidime-clavulanic acid combination was taken as positive.

\section{Determination of multidrug resistance in bacterial isolates}

The isolates were defined as multidrug resistant if they were resistant to two or more than two class of antibiotics tested or microorganisms which showed resistance to at least one antibiotic among three or more antimicrobial categories (Meligy et al., 2015; Clinical Laboratory Standard Institute, 2010; Jasuja et al., 2017).

\section{Statistical analysis}

Data so collected was analyzed by using Software Statistical Package for Social Sciences (SPSS) 16. The statistical testing was carried out by employing chi square test.

\section{Ethical issue}

Ethical approval was obtained from Institutional Ethical Committee before the beginning of the study.

\section{Results and Discussion}

In our study we observed a total of 101 ETAs culture positive patients in which 56 were males and 45 were females. Out of 101 positive cases, $44(43.56 \%)$ belonged to 0 to 5 years of age followed by $31(30.69 \%)$ in 6 to $15 \mathrm{yrs}, 14(13.86 \%)$ in 16 to $25 \mathrm{yrs}, 8(7.92 \%)$ in 26-50yrs and $04(3.96 \%)$ in $<50 y r s$ age group. The age and sex demographical distribution pattern is shown in table 1 .

In 101 culture positive isolates, the frequency of isolation of Gram-negative bacteria ( $n=79$, $78.2 \%)$ was found to be higher than $(n=22$, $21.7 \%$ ) Gram-positive bacteria. The most common micro-organism isolated was Klebseilla pneumoniae 28 (27\%) followed by Pseudomonas aeruginosa $22 \quad$ (22\%), Acinetobacter spp. 11 (11\%), E. coli 9 (9\%), Citrobacter $5(5 \%)$ and Proteus 4 (4\%). The most frequently identified micro-organism in Gram positive bacteria was CONS 12 (12\%), subsequently followed by $8 \quad(8 \%)$ Staphylococcus aureus and 2 (2\%) Streptococcus spp (Figure 1).

Antibiotic resistance pattern among Gram positive bacteria: Gram positive bacteria were highly resistant to penicillin $G(86 \%)$ which included S. aureus and CoNS which showed $87 \%$ and $91 \%$ resistance respectively.

Staphylococcus aureus showed high degree of resistance to gentamicin $(75 \%)$ followed by clindamycin and amoxicillin clavulanic acid (62\% each), co-trimoxazole (50\%), ampicillin sulbactam and azithromycin (37\% each) and least resistance was found to teicoplanin and linezolid $(12.5 \%)$ followed by vancomycin, ciprofloxacin and tetracyclin (25\% each). CoNS were highly resistant to clindamycin (83\%) followed by gentamycin (75\%), amoxicillin clavulanic acid (66\%), tetracycline $(58 \%)$ ciprofloxacin $(50 \%)$, co-trimoxazole and azithromycin $(41 \%)$ and least resistance 
was found to teicoplanin (8\%) followed by vancomycin (16\%), ampicillin-sulbactam $(16 \%)$. However no resistance was detected to linezolid among CoNS. (Table 2) shows the antibiotic resistance pattern of isolates in Gram positive bacteria.

Methicillin resistance was observed in $61 \%$ of Staphylococcus aureus isolates and 53\% of CoNS isolates by the Cefoxitin disc diffusion method.

Antibiotic resistance pattern among Gram negative bacteria: The highest resistance were found in two major beta-lactam antibiotic groups including $3^{\text {rd }}$ generation cephalosporins and Penicillin group antibiotics, the least resistance was found to carbapenems and polymyxin B.

Klebsiella pneumoniae showed maximum resistance to third generation cephalosporins including cefotaxime (85\%), cefoperazone (82\%), ceftazidime (75\%). Subsequently followed by amoxicillin clavulanic acid (68\%), gentamicin (57\%), ampicillin and tetracycline (50\% each), ciprofloxacin (46\%), tobramycin $(42 \%)$ and least resistance to imipenem (7\%) and polymyxin B (39\%). Ecoli was highly resistant to cefotaxime (77\%), cefoperazone and amoxicillin clavulanic acid (66\% each). ceftazidime, piperacillin/ tazobactam and gentamycin (55\%) showed similar resistance pattern and lowest resistance were found in imipenem and polymyxin B (33\%). In Citrobacter spp. high resistance was found in cefoperazone (80\%) and similar resistance to cefoperazone, cefotaxime and gentamicin $(60 \%)$ and least to imipenem, ampicillin and tobramycin (20\% each). Proteus spp also showed high resistance to cefoperazone and ceftazidime (75\%) equally and least to imipenem (20\%). In non-lactose fermenters Acinetobacter spp showed high resistance to cefoperazone (90\%), ceftazidime $(81 \%)$, cefotaxime $(72 \%)$ and amoxicillin clavulanic acid $(81 \%)$ and least to imipenem (18\%) and polymyxin B (27\%). Similarly Pseudomonas aeruginosa also showed high resistance to cefoperazone and amoxicillin clavulanic acid (68\%), and least to imipenem (4\%) and polymyxin B (18\%). Most of the Gram negative organisms were highly resistant to amoxicillin and cephalosporins and none of the isolates were found resistant to colistin (Table 3).

The Gram negative isolates (79) were tested for ESBL production, among which 47 (59\%) were ESBL producers.

The percentage of beta lactamase producing organisms is as follows: Klebsiella pneumoniae $17 / 28 \quad(60.71 \%), \quad$ E. coli 5/9(55.55\%), Citrobacter spp. 3/5(60\%), Proteus spp. 2/4 (50\%) and in non-lactose fermenters (NLFs), Acinetobacter baumannii $7 / 11$ (63.63\%), Pseudomonas aeruginosa $12 / 22(54.54 \%)$.

Respiratory tract infections due to the mechanical ventilation or tracheal intubation are the most important and leading cause of morbidity in ICU, NICU and emergency. The key treatment is early identification of the causative microbial agents, antibiotic sensitivity profile and administration of selected antibiotics. Endotracheal tubes are susceptible to impaction and attachment of micro-organisms therefore it is important to be aware of the relevant factors and responsible organisms to take prompt action.

In this study, 101 positive microbial cultures obtained from the ETAs or tracheostomy cases were considered for the identification of pathogens and their antibiotic resistance pattern. In our study, of all the culture positive patients 56 were males and 45 were females. Joseph et al., also reported quite similar gender ratio in his prospective observational study (Joseph et al., 2010). 
Figure - 1: Isolated microorganisms from intubated patients of ICU, CCU, NICU, PICU at BRD Medical College Gorakhpur.

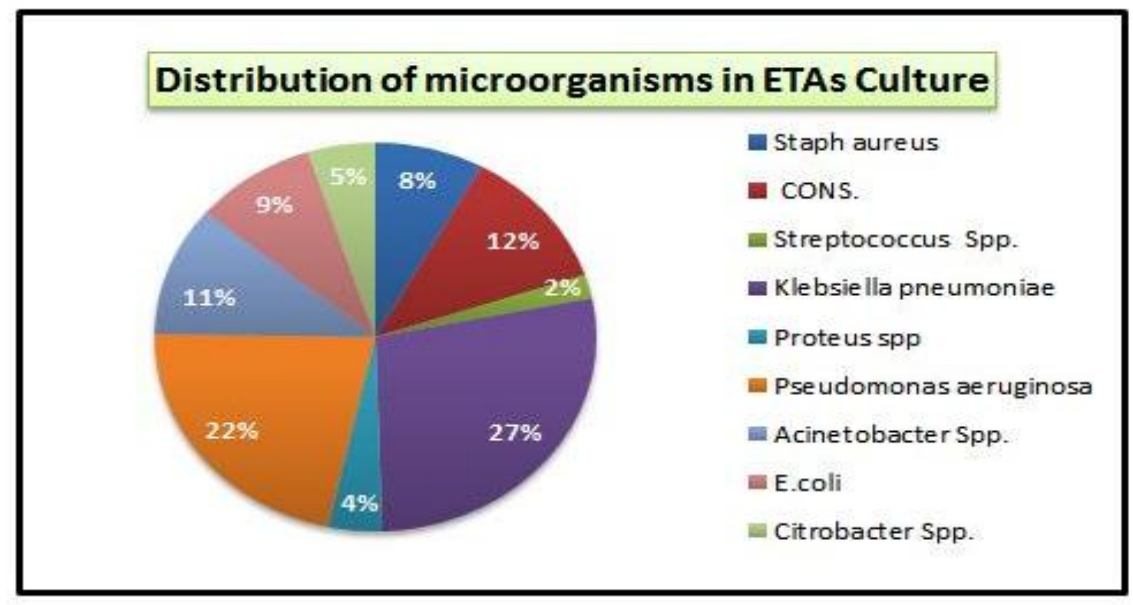

Table.1 Demographic distribution of ETA positive culture patients
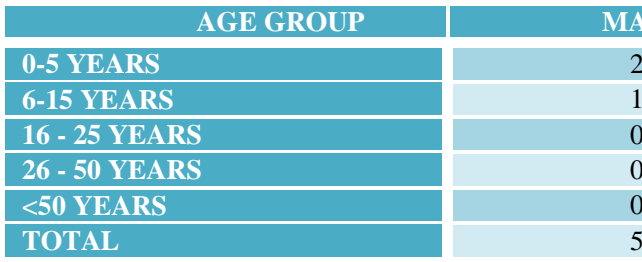

\begin{tabular}{|c|c|}
\hline MALE & FEN \\
\hline 28 & \\
\hline 13 & \\
\hline 08 & \\
\hline 05 & 03 \\
\hline 02 & \\
\hline 56 & 02 \\
\hline
\end{tabular}

\begin{tabular}{|c|c|}
\hline EMALE & TOTAI \\
\hline 16 & 44 \\
\hline 18 & 31 \\
\hline 06 & 14 \\
\hline 02 & 08 \\
\hline 45 & 04 \\
\hline
\end{tabular}

Table.2 Antibiotic resistance pattern among Gram positive bacteria

\begin{tabular}{|c|c|c|c|c|c|c|c|c|c|c|c|c|}
\hline MICRO. & AMC & AZM & CI & PG & TE & COT & $\mathbf{L Z}$ & TP & VM & $\mathbf{A M}$ & CM & GEN \\
\hline $\begin{array}{l}\text { Staphylococcus } \\
\text { aureus }(\mathrm{n}=\mathbf{8})\end{array}$ & 5 & 3 & 2 & 7 & 2 & 4 & 1 & 1 & 2 & 3 & 5 & 6 \\
\hline CONS. $(\mathrm{n}=12)$ & 8 & 5 & 6 & 11 & 7 & 5 & $\mathbf{0}$ & 1 & 2 & 4 & 10 & 9 \\
\hline $\begin{array}{l}\text { Streptococcus } \\
\text { spp. }(\mathrm{n}=\mathbf{2})\end{array}$ & 1 & $\mathbf{0}$ & 1 & 1 & 0 & 1 & $\mathbf{0}$ & 1 & 1 & $\mathbf{0}$ & $\mathbf{0}$ & 1 \\
\hline Total $(n=22)$ & 14 & 8 & 9 & 19 & 9 & 10 & 1 & 3 & 5 & 7 & 15 & 16 \\
\hline
\end{tabular}

Table.3 Antibiotic resistance pattern among Gram negative bacteria

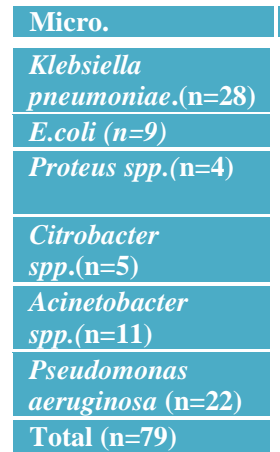

\begin{tabular}{|c|c|c|c|c|c|c|c|c|c|c|c|}
\hline AMC & AK & CFS & CE & CAZ & PT & $\mathrm{TE}$ & IPM & CI & TB & GEN & PB \\
\hline 20 & 14 & 23 & 24 & 21 & 15 & 14 & 2 & 13 & 12 & 16 & 11 \\
\hline 6 & 3 & 6 & 7 & 5 & 5 & 4 & 1 & 4 & 2 & 5 & 3 \\
\hline 2 & 1 & 3 & 2 & 3 & 2 & 1 & 1 & 3 & 2 & & 2 \\
\hline 2 & 1 & 3 & 3 & 4 & 3 & 2 & 1 & 2 & 1 & 3 & 2 \\
\hline 9 & 5 & 10 & 8 & 9 & 6 & 6 & 2 & 6 & 5 & 7 & 3 \\
\hline 15 & 8 & 16 & 15 & 13 & 12 & 12 & 1 & 14 & 6 & 8 & 4 \\
\hline 54 & 31 & 61 & 59 & 55 & 43 & 39 & 8 & 42 & 28 & 42 & 25 \\
\hline
\end{tabular}


We found the frequency of isolation of Gramnegative bacteria to be higher than Grampositive bacteria, similarly Abdollahi et al., and Ferreira et al., had also shown high frequency of Gram-negative microorganisms isolated in ETAs culture (Ferreira et al., 2016; Abdollahi et al., 2013). In our study, the most common micro-organism was Klebseilla pneumoniae among all isolates followed by Pseudomonas aeruginosa, Acinetobacter spp., E. coli, Citrobacter spp. and Proteus. The most frequently identified micro-organism in Gram positive bacteria was CONS subsequently followed by Staphylococcus aureus. This finding is in accordance with the results of Bassant et al., who found that the most prevalent isolates in quantitative ETA cultures were GNBs (77.3\%) such as Klebsiella, Acinetobacter, Pseudomonas and Proteus spp., whereas Gram- positive organisms accounted for $22.7 \%$ represented by CONS (Meligy et al., 2015). Amini et al., also conducted a descriptive study showing similar distribution of isolated microorganisms (Amini et al., 2009).

Our studies as well as the other ones in similar settings confirm that Klebsiella pneumonia, P.aeruginosa and CoNS are among the most 3 prevalent isolated organism from endotracheal tube aspirate (Jasuja et al., 2017, Abdollahi et al., 2013; Amini et al., 2009).

It is important to understand that although there are minor variations in relative frequency of obtained organisms, different studies have reported varying sources of isolations upon different geographical area and applied methodology.

In the current study, the frequency of polymicrobial infection was present in 7 $(6.93 \%)$ patients. Several researchers estimated that polymicrobial infections accounted for $20-60 \%$ of patients (Wilson et al., 2012).

Gram positive bacteria were highly resistance to penicillin $\mathrm{G}$, gentamycin and clindamycin least resistance were found to teicoplanin, linezolid and vancomycin. Methicillin resistance was observed in $61 \%$ of Staphylococcus aureus and $53 \%$ in CoNS. In this respect, our study was quite similar to the other studies, Bassant et al., reported methicillin resistance was detected in $60 \%$ of GPC isolates. All isolates were $80 \%$ resistant to penicillin $\mathrm{G}$ and $100 \%$ resistant to clindamycin. Another study Abdollahi et al., claimed that $84 \%$ Gram positive organism including Staphylococcus aureus and CoNS were resistant to cefoxitin and showed increasing frequency of multidrug resistance in GPC which represent the emergence of MDR in MV patients (Meligy et al., 2015, Abdollahi et al., 2013).

Antibiotic resistance pattern among Gram negative bacteria: the highest resistance were found in two major beta-lactam antibiotics including $3^{\text {rd }}$ generation cephalosporins and Penicillin group of antibiotics. All the lactose fermenting Enterobacteriacae were highly resistant to cephalosporins including cefoperazone, cefotaxime and ceftazidime similarly non lactose fermenters Pseudomonas aeruginosa and Acinetobacter spp also showed high resistance to cefoperazone and amoxicillin clavulanic acid and least to imipenem and polymyxinB.

Among Gram negative isolates tested for ESBL production; 50 out of $79(63 \%)$ were ESBL producers. Our findings were similar when compared to the Kiran Tandia et al., and Abdollahi et al., who reported high degree of resistance in cephalosporins and Penicillin group of antibiotics in their studies (Tandia et al., 2015, Abdollahi et al., 2013).

The micro-organisms that have been documented to colonize ETTs and grow in the 
form of a biofilm are numerous, including the multidrug-resistant bacteria MRSA and Gram-negative bacilli such as $K$. pneumoniae, E. coli, $P$. aeruginosa and Acinetobacter spp. Therefore, making effective antibiotic policy directed at reducing such colonization could help to reduce the rates of antibiotic-resistant infections.

Our observations provide useful information regarding the microbiology of respiratory infection occurring in intubated patients. In the present study, antimicrobial resistance rate was alarming in the culture of ETA isolates obtained from MV patients. This study also highlighted the importance of using empirical antimicrobial therapies to target multidrugresistant pathogens at early-onset VAP. Therefore, ETA culture provides accurate and reliable information in respect to early treatment strategy, swift administration of antibiotics as per susceptibility results and should be routinely included as a part of management protocol.

Thus ET tip cultures could be a useful adjunct to the ATS (American Thoracic Society) strategy for ensuring appropriate treatment of intubated patients and in the context of a bundled approach to the prevention of ET tip infections.

\section{Acknowledgement}

The author is immensely thankful to Dr. C. Upadhyay, Professor, Department of Microbiology, GSVM Medical College, Kanpur for his support and guidance to complete the study.

\section{Source of Support}

Nil

\section{Conflict of Interest}

None declared.

\section{References}

Abdollahi A, Shoar S, Shoar N. Microorganisms' colonization and their antibiotic resistance pattern in oro tracheal tube. IRAN. J. MICROBIOL. (2013), Vol. 5, No. 2 102-107.

American Thoracic Society, Infectious Diseases Society of America. Guidelines for the management of adults with hospitalacquired, ventilator-associated, and healthcare-associated pneumonia. Am J Respir Crit Care Med. (2005) 171: 388416.

Amini M Javanmard A, Davati A, Azimi G. Bacterial colonization in tracheal tubes of ICU patients. Iranian Journal of Pathology. (2009) 4:123-127.

Chandra Mouli HC, Nagaraja M, Chandra A, Kalawat U. Endotracheal tube tip culture in post-operative respiratory infections in open heart surgery patients: a one year prospective study. J Clin Sci Res (2016)5:214-20.

Chastre J, and Fagon JY. Ventilator-associated pneumonia. Am J RespirCrit Care Med (2002) 165: 867-903.

Chevret S, Hemmer M, Carlet J et al., Incidence and risk factors of. Pneumonia acquired in intensive care units. Results from a multicenter prospective study on 996 patients. European Cooperative Group on Nosocomial Pneumonia. Intensive Care Med (1993) 19:256-64.

Chevret S, Hemmer M, Carlet J, Langer M. Incidence and risk factors of pneumonia acquired in intensive care units. Results from a multicenter prospective study on 996 patients. European Cooperative Group on Nosocomial Pneumonia. Intensive Care Med (1993) 19:256-264.

Clinical Laboratory Standard Institute (CLSI) Performance Standards for Antimicrobial Susceptibility Testing; 20th in formational Supplement; Clinical and Laboratory Standard Institute, Wayne, Pennsylvania, USA; 2010, M100S20.

Clinical Laboratory Standard Institute. Performance standard for antimicrobial 
disk susceptibility tests; approved standard $-20^{\text {th }}$ Ed. Supplement/ M2 - A9 (2006) 26:1.

Collee JG, Fraser A.G, Marmian B.P et al., (1996) Mackie and McCartney Practical Medical Microbiology. Pearson Professional Ltd $14^{\text {th }}$ edition ISBN: 0443047219.

Depuydt P, Benoit D, Vogelaers D, Claeys G, Verschraegen G, Vandewoude $\mathrm{K}$, et al., Outcome in bacteremia associated with nosocomial pneumonia and the impact of pathogen prediction by tracheal surveillance cultures. Intensive Care $\operatorname{Med}(2006)$ 32:1773-81

Diaz E, Planas K, Rello J. Infection associated with the use of assisted-ventilation devices. EnfermInfecc MicrobiolClin (2008) 26: 465-470.

Donald E. Craven, Karin I. Hjalmarson. Ventilator-Associated Tracheobronchitis and Pneumonia: Thinking Outside the Box. Clinical Infectious Diseases (2010) 51(S1):S59-S66.

Ferreira T.O, Koto R.Y, Leite G.F.C et al., Microbial investigation of biofilms recovered from endotracheal tubes using sonication in intensive care unit pediatric patients, The Brazilian Journal of Infectious Diseases, (2016) 20: 5; 468475.

Fortaleza CM, Abati PA, Batista MR, Dias A. Risk factors for hospital-acquired pneumonia in nonventilated adults. Braz $J$ Infect Dis (2009) 13: 284-288.

Haley RW, Hooton TM, Culver DH, Stanley $\mathrm{RC}$, Emori TG, Hardison CD, et al., Nosocomial infections in U. Shospitals, 1975-1976: estimated frequency by selected characteristics of patients. Am J Med (1981) 70: 947959.
Infection Prevention Endotracheal Tube. https://www.bactiguard.se/en/our products/endotracheal-tube.

Jasuja K, kumar, Singh A.K, Kumar A et al., Emergence of Multi-drug Resistant Bacterial isolates causing Blood stream infections in paediatric patients at a tertiary care hospital, British $\mathrm{j}$ of pharm and med Sci.(2017) 2:2; 298-407.

Joseph N.M, Sistla S, Dutta T.K et al., Ventilator-associated pneumonia: role of colonizers and value of routine endotracheal aspirate cultures. International Journal of Infectious Diseases 14 (2010) e723-e729.

Kollef M.H, and Fraser V.J. Antibiotic Resistance in the Intensive Care Unit. Ann Intern Med. (2001)134:298-314.

Meligy B, Khairat S, Sayed A, Azmy M, Ismail D.K, Yahia S. A study of biofilm on endotracheal tubes in pediatric intensive care unit. Kasr Al Ainy Medical Journal (2015) 21:87-93.

Nseir S, Favory R, Jozefowicz E et al., Antimicrobial treatment for ventilatorassociated tracheobronchitis: a randomized controlled multicenter study. Crit Care (2008) 12: R62.

Tandia K, Wadhwani J. L., Sharma M. A Clinical Study of Pattern of Microbiological Colonization of Endotracheal Tube Aspirate on Mechanically Ventilated Patients. International Journal of Science and Research (2015): 2319-7064.

Wilson A, Gray D, Karakiozis J, Thomas J. Advanced endotracheal tube biofilm stage, not duration of intubation, is related to pneumonia. J Trauma Acute Care Surg (2012)72:916-923.

\section{How to cite this article:}

Vandana Upadhyay, Ankur Kumar, Jayesh, Amresh Kumar Singh. 2018. A Study of Microbial Colonization and their Antibiotic Resistance Pattern in Endotracheal Aspirate Cultures in Intensive Care Unit Patients at a Tertiary Care Hospital of Eastern UP, India. Int.J.Curr.Microbiol.App.Sci. 7(04): 2298-2306. doi: https://doi.org/10.20546/ijcmas.2018.704.263 